\title{
Index
}

Aaruul, see Qrut

Abū Hāmid 114

Acorns, as Korean food 283

Afghan language 73

Afghanistan $1,7,9,28,34,40,73,75,90,175^{-}$ $176,177,179,204,206-219$

Bread 207-208

Dairy products 215

Drinks 216

Festivals 207

First agriculture 75

Flavorings $\quad 215-216$

Food influences 219

Food words 215

Fowl 215

Kebabs 214-215

Markets 218

Meat stewed with fruit $\quad$ 212-213

Pasta 208

Rice 209

Sweets $\quad 216$

Types of pilau 209-210

Weddings $\quad 217-218$

Agriculture, advance of, spread into Central Asia $\quad 53-55,74-75$

Ai Khanum 90

Al-Bīrūnī (973-1048), 48, 116, 122

Al-Khwārizmī $\quad 116$

Alcohol 3, 199

Alcoholic drinks $\quad$ 191, 258-261, 284

Korean 284

Mongolian 258-261

Ale 57,151

Millet ale $\quad 151$

Rice ale (terracina, "rice wine") $\quad 151$

Alexander the Great $\quad$ 90, 116-117

Alfalfa $\quad 42,57,88,98$

Allsen, Thomas $\quad 22,143$

Almaty 32,60

Almonds 57, 106, 125, 148, 238, 265

In Ibn Sīnā's Canon 125

Wild species, in Mongolia 265

Altai Mountains $\quad 7,8,12,59,71$

Altaic languages $\quad 70$
Ammianus Marcellinus 99-100, 129

On Hun 99-100

Amu Darya 9, 28, 34

Amur-Ussuri 171

Animal-style art, spread of 80

Animal life, overview $\quad$ 21-29

Animals, domestication $\quad 63,65$

Effects of domestication 63

History of domestication in Eurasia 63

Self-taming 65

Anise 104

An Lushan 安祿山 Rebellion 105

Apples 18, 32, 57, 6o, 61, 94, 112, 148, 174, 181, $205,236,238$

Apricots $32,57,94,106,125,174,205,235^{-}$ 236,238

Aqsakal 126

Aquaculture 95

Aral Sea 9, 18, 27-29, 35

Arabian spices 137

Arabs 88, 104

Conquest of western Central Asia $\quad 88$, 104

Araq ('araq) 51, 198. See also Arkhi

Area of Central Asian nations 31

Aristotle 123

Arkhi 51, 153-154, 285. See also Araq

Armenian language $\quad 72$

Arseniev, v. K. 175

On Tungus 175

Ash 193

Ashak 208

Āthārva Ahyyā' 144

Avars (Rouran 柔然) 44-5

Avicenna, see Ibn Sīnā

Azerbaijan food 250-254

Food proverbs $\quad 25^{2}$

Spices and flavorings $\quad 251$

Sweets 253-254

Azeri, language $\quad 251$

Babur $\quad 161$

Bactria $\quad 90-91$

Bagel 184-185 
Baghdad, Mongol conquest of 132

Baklava 231

Bal 151

Balancing diet 119

Balpo Soup 193

Bamboo 125

Bamiyan 104

"Barbarian nomad," myth of 44

Barberries, in Iran 220

Barfield, Thomas 38

Barley $31,49,57,59,75,77,81,112,120,148$, $173,174,178,181,235,279,283$

Broth, in humoral medicine 120

In Afghanistan bread 206

Spread into Central Asia 75

Spread into China 77

Barley Samosa Noodles $\quad 188$

Barley Soup $25^{2}$

Bashkirs 173

Batu 131-132

Ba'urchi 143

Baysursak 246-248

Bean curd (doufu 豆腐) 48

Beans 174, 176, 180, 284

Broad 105

Fava 235

Green (fresh) 180

Hyacinth 174

In kimchi $\quad 284$

Small dry green $\quad$ 174. See also Mung beans

Beansprouts 284

Beetroot 174

Beijing 北京 130, 134

Founded by the Mongols 130

Bencao Gangmu 本草綱目 (Chinese herbal) 123

Birch beer 112

Birds, hunting $\quad 25-26$

Black Death 25

And rodents 25

Black-eyed peas 223

Black gram 125

Black kumiss 149

Blood, as food 195

Bolad 143-144

"Booze," 191

Botai Culture, horse meat $\quad 76$
Boza 191, 270. See also "Booze"

Bread 56, 119, 182-184, 206-208, 263, 279

In Afghanistan 206-208

In humoral medicine 119

In Mongolia 263

Sacredness $\quad 183$

Britain 29

British 175

Influence on food 205

Bronze Age $\quad 52,76,81$

Buckwheat 94, 283

Buddhism 125

Buddhist travelers to China 111

Bukhara 3, 73, 162, 204, 221

Burani, eggplant dishes 212

Buran, Princess 212

Burma 133

Butter 148, 197

Butter-making 197

Buudz 263

Byzantine Empire $\quad 45,88$

Ca'adai Qanate 134

Cabbage 181

Chinese cabbage $\quad 284$

Camels, Bactrian $\quad 41,48,66,69,78,173,192$, 264, 269

In early Xinjiang 新疆 78

Camels, dromedary 69

Caracomos 151

Caraway, black 62

Caravan trade 2

Cardamom 49, 6o, 125, 181, 202

Large cardamoms 202

Carrots 49, 174, 181, 211, 28。

Varieties 211

Caspian Sea 9, $5^{2}$

Castoreum 146

Cassia 95, 148

Cats 280

Cattle $41-42,49,65,69,78,173,181,192,264$, $268,280,285$

Beef in Korea 285

In early Xinjiang 78

Central Asia $\quad 2,4,22,38,74,39-40,82-84$, 88-89, 121, 126, 162, 173-179,

Center of Galenic Medicine 121

Food 2 
Food un-Sinicized $\quad 178$

Foodways and religion $\quad 82$

Globalization $\quad$ 178-179

Governance 126

Herding 38

Islam $\quad 83-84$

New World foods $176-177$

19th and 2oth century food 174

Nomads 39-40

Post-Mongol governance 173

"Race," 74

Recent history $\quad 162,177^{-178}$

Slaving $\quad 88-89$

Writings on hunting 22

Chaikhana (teahouse) 222

Champa 133

Chang'an 長安 6

Ethnic communities 6

Chang Chun 長春 148

Chariots, origin and spread to China 76,77

Charpentier, C. J. 218

Chen, Qiang 170

Cherry Concentrate $\quad 251$

Cherries $32,174,238$

Chestnuts 94,283

Chickens 69, 196

In Afghanistan 215

Chickpeas 54-56, 235

Chiles 49, 162, 174, 176, 202, 205, 236, 281, 284

China 1, 12, 37, 47-48, 54-56, 77-80, 86-88, $92,95,102,107,132-136,178-179,280-281$, 379

And Central Asia 77

Animals from the West 280

Archery 79

Early contacts 79

Early dynasties $\quad 77,87-88$

Economic center of world $\quad 134$

Food flows 95

Foods coming to China with Eurasian connections 107, 379

Gansu 甘肅 corridor 77

Greek influences 92

Han 漢 Dynasty 88

Han Dynasty and Central Asia 88

Herbal literature 102

History of agriculture $\quad 54,56$

Horse 88
In Mongol Empire 133

Merged into Central Asia 92

Military technology 79

Mongol Yuan 元 Dynasty 86

Near Eastern Spices 280

New World crops $\quad 281$

Western grains other than wheat 379

Qijia 齊家 Culture 77

Shiba 寺洼 Culture 77

Stimulus diffusion $\quad 54$

Tombs 80

"Western Regions," 86

Western vegetables 280

Writing 78

Chinese chives (garlic chives) 95, 96, 200, 202, 231

Chinese foods of Central Asian background 271-281

In Ming 明 Dynasty 271-279

In modern times $\quad 279-281$

Chinese language $\quad 70,78-79$

Early Indo-European loan words $\quad$ 78-79

Chinese pulsing 148

Chocolate 281

Chopsticks 97

Chuqmin 207-208

Cinggis-qan $\quad 35,47,87,108,110,115,126-129$

His starvation food in childhood $127-128$

Cinnamon 49, 62, 125, 181, 201

Citrus fruit, from China $\quad$ 107-108

Clavijo, Ruy Gonzoles de 141

Climate 12-13, 18-20

History $\quad 12-13$

Cloves $\quad 62,125,181$

Coconut 125

Collective management 40

Confucianism 77

Congee, in Korea 283

Cooking utensils $\quad 202-203$

Copper smelting 75

Core and periphery 87

Coriander $49,57,62,104,105,181,201,28$ o

Cosmas Indicopleustes 110-111

Cotton 28, 31, 62, 88, 125, 224

Cowpeas 107

Cucumber 49, 105, 181, 200

Cumin 49, 57, 62, 104, 106, 181, 201, 280 


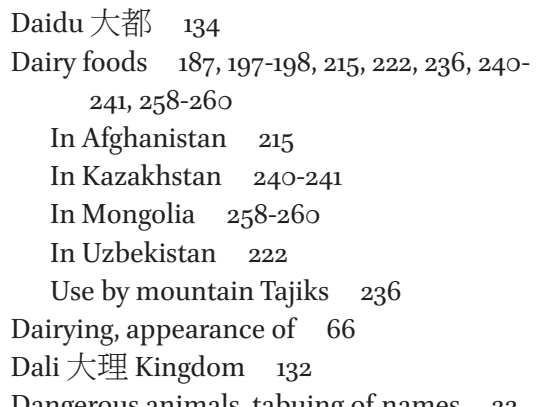

Dangerous animals, tabuing of names 22

Daoism 77

Dari language 73

Dastarkhan 239

Dates 106, 115, 220

In Iran 220

Deer Stone-Khirigsuur complex 66

Dersu the Hunter 175

Deodar 125

Descent groups and resources 127

Dietary Therapy, Galenic $\quad$ 118-120

Dill 202

Dioscorides, Pedanios $\quad 117,120,124$

Distillation $\quad$ 51, 164-169, 280, 285-286

Gaoliang 高粱 280

In Korea 285-286

Milk liquor $\quad 51$

Dock (herb) 202

Dogs, Central Asian breeds $\quad 54,63-64,285$

As food, Korea $\quad 285$

Domestication of the dog 54, 63

Domestication $\quad 53-55$

Defined 54

In prehistory 53

Dong'an 233, 257

Donkeys 41-42, 173

Dopiaza 215

Dormice, as food 150

Dragon fruit 281

Duck (domestic) 95

Dumplings $\quad 95,181,184-190,263,270,279,285$

In Korea $\quad 285$

In Mongolia 263

Kalmyk 270

Dungan, see Dong'an

Dunhuang 敦煌 oasis 6, 93

Dupree, Louis 40, 206-219

Dūqū 190
Dzungar 29

Dzungaria 8

Eastern Iran, food 219

Eggplant 49, 148, 212

Embelia 125

Enin 186

Eurasian Heartland $\quad$ 1, 7

Eurasian Heartland, agriculture $\quad 30-32,38$

And environment $\quad 30-31$

And livestock 38

Eurasian Heartland, and conflict

Eurasian Heartland, animals 21

Eurasian Heartland, birds 24-26

Eurasian Heartland, bread $\quad$ 182-184

Nan 182-183

Eurasian Heartland, cities 37

Urban sprawl 37

Eurasian Heartland, climate $\quad$ 19-20

Climate change $\quad$ 12-13

Eurasian Heartland, contemporary foodways $180-182$

Eurasian Heartland, cooking utensils 202

Eurasian Heartland, crossroads 48

Eurasian Heartland, desert woodlands $\quad 15$

Eurasian Heartland, ecological system $\quad 51$

Eurasian Heartland, environmental problems 26-27

And religion 26-27

Eurasian Heartland, foods $\quad 32,49-51,190-192$, 197-203

Dairy foods $\quad$ 197-198

Drinks 198

Grain foods 190-192

Overall views of $\quad 49-51$

Sour preferred 202

Spicing 202-203

Sweet fruits and berries preferred 200201

Sweets 200

Vegetables 199-200

Eurasian Heartland, foodways, sources of change 204-205

Eurasian Heartland, grasslands 37

Eurasian Heartland, herding $\quad 38-39,46$

Types 46

Eurasian Heartland, history before the Mongols 86 
Eurasian Heartland, hunting $\quad 21-24$

Eurasian Heartland, irrigation 32

Eurasian Heartland, languages $\quad$ 70-73

Chinese 72

Dari 73

Farsi 72

Ket 71

Russian 73

Tajik 73

Tibetan 71

Tokharian 71

Turkic 72

Xianbei鮮卑 70

Xiongnu 匈奴 70

Eurasian Heartland, linguistic Stalinism 73

Eurasian Heartland, linking area $\quad 48$

Eurasian Heartland, little Chinese influence 96

Eurasian Heartland, major crops, animals, advance of 54

Eurasian Heartland, major medical centers in past $121-122$

Eurasian Heartland, meat $\quad$ 192-196

Cooking 192-195

Sources 192-195

Eurasian Heartland, medicinal herbs $\quad 18$

Eurasian Heartland, mountains, mountain landscapes 8-9, 15

Eurasian Heartland, mythology 81

Eurasian Heartland, nationalism 73

Eurasian Heartland, nations $39-40$

Eurasian Heartland, noodle $\quad$ 184-185

Eurasian Heartland, oases 35

And agriculture $\quad 35$

And agriculture and irrigation $\quad 35$

Eurasian Heartland, origins of civilization 74-76

Eurasian Heartland, pastures 13-14

Eurasian Heartland, penetration by Russia 172

Eurasian Heartland, Qing 清 conquests 172

Eurasian Heartland, rain $\quad 12$

Eurasian Heartland, religions 82

Eurasian Heartland, riparian forests 15

Eurasian Heartland, rivers $\quad$ 9, 12, 32, 34-35

Eurasian Heartland, small mammals 25

Eurasian Heartland, steppe soils 37

Eurasian Heartland, tree crops 32
Eurasian Heartland, vegetation 13

Falck, Johan Peter 173

On Central Asian foods and foodways 173

Falconry 21-22, 173

Farsi language $\quad 72,93,204,212$

Farsi food words in Central Asia 93, 204

In Afghanistan 212

Faxian 法顯, monk 111

Feasting 55

Feijoa 254

Fennel 106, 280

Fenugreek 104

Fermented mares' milk, see Kumiz

Ferghana, Manti 187

Ferns, in Korean food 284

Fertile Crescent 57

Festival foods 217, 237

In Afghanistan 217

Among mountain Tajiks 237

Figs 106, 115

Fish, food species of Central Asia $\quad 196$

Fish oil 112

Five Snouts $\quad 264$

Flax 106

Fletcher, Joseph 127

Food borrowings from China $\quad$ 96-97

Galen 102, 116, 117, 118, 120-121, 145, 147

And Dioscorides, Arabic translations 120-121

Food coding of 102

Medicine 118

Gansu 93

Gao Lian 高濂 $\quad$ 172, 271-277

On Ghee 273

On Halwa 271

Recipe for Black and Moist $\quad 276$

Recipe for Comp-print Crisps $\quad 275$

Recipe for Crisp Cookies 277

Recipe for Goat Marrows $\quad 276$

Recipe for Making Sugar Syrup $\quad 272$

Recipe for Manchu Candied Fritters 274

Recipe for Nest of Threads 275

Recipe for Oil Harmonized with Flour 273 
Recipe for Pepper and Salt Cookies $\quad 276$ 277

Recipe for Pine-nut Cookies 273

Recipe for Pine-nut Hailuo 海羅 273

Recipe for Puffed Buckwheat 275

Recipe for Roasted Flour $\quad$ 272-273

Recipe for Saboni $\quad 276$

Recipe for Slices with Mint 275

Recipe for Snow White Shortbread 274

Recipe for White and Moist $\quad 274$

Recipes for Wind-Dissolved Cakes $\quad$ 277278

Recipe for Yellow and Moisturized Halwa 274

Sweets 172

Gaoliang (Chinese liquor) 6o

Garlic 49, 280

Garlic chives, see Chinese chives

Geography, physical 8, 9

Georgian language 72

Ger (yurt) 43

Ginger 49, 182, 202

Ginseng 175

Globalization $\quad 204$

Goats $41-42,49,64,173,181,192,220,264$, 280

Göbekli Tepe $\quad 55-56$

Gobi 44, 257

Goens, Benedikt on food 163

Gog and Magog 110-111

Goji berries 280. See also Lycium

Golden Horde 134

Cities of the Volga 140

Goose (domestic) 95

"Grains of Paradise," 6

Grapes $31,57,60,81,88,174,181,205$, 28 o

And raisins 31

Domestication 6o

Grasses and grasslands 13-15

"Great Game," 175

Greek language 72

Greeks 90 Influence on Central Asia 90

Grigor of Akanac 129

Grut 149, 152

Guava 281

Güyük-qan 131
Haider, Jamila, see Van Oudenhoven

Halva, see Halwa

Halwa 182, 201, 204, 256, 257, 262

In Uzbekistan 229

Halwåy Tar 278

Han Dynasty $\quad 88,89,97$

Han (nationality) supremacy 73

Hansen, Valerie $\quad$ 135, 137

Hayden, Brian, on feasting 55

Hazelnuts 112

Heaven 83

Helmand River 34

Herodotus 110

Hippocrates 116

Hippocratic-Galenic medicine $\quad$ 116-120, 142

Hö’elün-eke 127

Honey 112, 280

Honeybees 280

Horde, etymology 46

Horses 2, 23, 41-42, 47, 49, 54, 6o, 66-69, 76, 112,15 o, 173, 181, 192, 232, 238, 268, 28 o

And humans 68

Botai Culture and horse $\quad 67$

Domestication 2, 47, 66-68

Kazakh consumption $\quad 238$

Meat $\quad 66-67$

Milk 67

Origins, and Przhevalsky's Horse 66

Sacrificed in medieval Russia 112

Sausages of horse meat (kazy) 150, 173, 232

Hu Sihui 忽思慧 24, 159

Huihui Yaofang 回回藥方 (Chinese medical encyclopedia) 123, 144-147, 159-161

Hüle'ü $\quad 132,133$

Humors, of Galenic medicine $\quad$ 118-119

Huns 98-101

And raw meat 99

Hunting 21-29

Hunting Eagles 21-22

Huntington, Ellsworth 37

Huntun 餛飩 186

Ibn Battuta, on foods 196

On foods of later Uzbekistan $\quad$ 221-222

Ibn Butlān $\quad 125$

Ibn Faḍlān 111-113

And food 112 
Ibn Khaldun 87

Ibn Sīnā (Avicenna) 48, 116, 122-125

Ilqanate 134

Inbalances 119

Indian Ocean, early accounts of 111

Indo-European language phylum 4, 70, 71- 72,78

And Uighurs 4

In early eastern Central Asia 78

Loanwords into Chinese 79

Spread into Central Asia 71-72

Inner Mongolia (Nei Menggu 內蒙古) 1, 15, $41,72,93,97,181$

Iran $30,36,52,111,148,181,219-221$

Eastern Iranian food $\quad 219-221$

Influences on food 181

Iranian Medicine $\quad 148$

Iranic languages 49, 70, 204

Irrigation $\quad 28-30,32$

'Isā 145

Isfahan 34

Işin, Priscilla Mary 254

Islam 48, 83-84, 104, 181

And Central Asian foodways 84

Conquests in Central Asia 104

Hanafi 83

Influence on foods $83-84,181$

Legal traditions of $83-84$

Itria 185

Japan, Mongol invasions 134

Japanese language $\quad 70$

Jebe, and Sübe'etei 131

Jia Sixie 賈思劦思 102

Jiaozi 餃子 186

Jenkinson, Anthony $\quad$ 141, 163

On food 163

Jerky 195

Jewish food in Uzbekistan 230-231

Jin 金 Dynasty 105, 109, 127, 130, 131

Jujia biyong shilei 居家必用事類, medieval

household book 155-189, 265

"Muslim Recipes," 155-189

Noodles 184

Recipes from 156-159

Jujube ("Chinese date") 94, 265

Juliana Anicia Codex 211

Jürchen 109
Juvayn̄̄ 128,130

Kababs 193, 214

In Afghanistan 214

Kabul 6o

Kaifeng 開封 128

Kalash 84 ; foodways 85

Kalmyk 165-166, 168, 268-271

Agriculture, plants grown $\quad 269$

Color symbolism of food 270

Dairy foods 270

Distillation $\quad$ 165-166, 168

Dumplings 270

Feasting 269-270

Food 268-271

Food intake 271

Food rules 270

Food tabus 271

Herding 268-269

Land use $\quad 268$

Pregnancy food rules 271

Tea 270

Tsatsal Ritual 271

Weddings 271

Karakorum Mountains $\quad$ 7,8

Karez $\quad 35-36$

Kazakh food $\quad 239-250$

Bread 246

Dairy 240-241

Jams, jellies and syrups $\quad 249-250$

Meat 239

Kazakh food proverbs $\quad 242-245$

Role of proverbs and song in foodways 242

Kazakhs 5, 21, 22, 173, 221

Kazakhstan $1,7,13,28,35,51,58,59,67,75$, $81,174,177,239,242$

Festive board 239

First agriculture 75

Russian and Korean food 242

Kazy 195

Kelley, Laura $\quad 251$

Khakass (Turkic people) 174

Khayyam, Omar 30, 192

Khazars 113

Khitan 81, 105, 108-109, 130

Khiva 204

Khorasan 1, 30, 86, 219, 220-221 
Typical spicing $\quad$ 220-221

Khuriltay $127,132,133$

Khwarazm (Khorasm, Khwarizm, Khwarazmia) $115,116,128,221$

Khwarazmian Empire 130

Khwarazm-shāh 131

Ki, Empress 282

Kimchi $51,284,286$

Kitābal-Tabīkh 113

Kitābal-Tibākha 113

Kitan 81

Kohlrabi 106, 280

Kokand 221

Kok chuchwara 228-229

Korea 49, 88, 178, 281-283

And interior Asia $\quad 283$

Differences from Central Asia 282

Influence at Mongol court $\quad 282$

Under the Mongols $\quad 281-282$

Korean food 177, 230, 281-286

Chinese influences on food $282-283$, 285-286

Congee $\quad 283$

Distillation 285-286

Finely cut meats $\quad 285$

Grain 283

In Uzbekistan 230

Influences from West $\quad 281$

Kimchi 284

Links to Central Asia 286

Makkŏlli and other drinks $\quad 285$

Meat 284

Noodle dishes $\quad 285$

Pickles 283-284

Pulgogi, conceptual stimulus for 285

Rice 283

Sauces $\quad 284$

Stuffed dumplings $\quad 282$

Korean language $\quad 70$

Kumiz (fermented mares' milk) 49, 76, 9o, $148,149,151,163,164,198,232$

Black kumiz $\quad 149,151$

Distilling for araq (arkhi) $\quad 164$

Kyrgyz 21, 238

Food 238

Kyrgyzstan 1, 174, 177, 234

Medicinal herbs, wild herbs for medicine and food 234
Lactase $\quad 75^{-76}, 197$

Genes 197

Lactobacillus $\quad 75^{-76}, 197,202,284$

Lactose, Eliminating $\quad$ 197-198

Tolerance and intolerance 75-76, 197

Laghman 190, 226, 231, 240, 255

Land connections, persistence of in Eurasia 141

Languages $\quad 70-73$

Language politics 73

Lattimore, Owen 38

Legumes 196

Lemon 107

Lemon juice 202

Lentils 56,235

Domestication of $\quad 56$

In Tajikstan 235

Lettuce 280

Li Shizhen 李時珍 123

Liao 遼 Dynasty $60,105,108$

Lily bulbs, as food 174

Lime 107

Little Ice Age 12-13.135

Livestock 38,42

And agriculture 38

Ecological diversification $\quad 42$

Long pepper, see Pepper, Long

Lop Nor $12,5^{2}$

Loptuq 257

Lotus 144

Lycium spp. 280

Mahmud al Kāshgarī 255

Mahmudov, Karim, on Uzbekistan food 231

Maize $31,162,174,175,176,180,206,281$

In Afghanistan bread 206

Manchu food names $\quad 172$

Mamluk Egypt 133

Mammals, hunting $\quad 26$

Mammoths 21, 53

Hunting 21

Manchuria, Turkish, Mongolian and Middle Eastern food words $\quad 171-172$

Manchus 170

Manila galleon $\quad 162,289$

Manta, see Manty

Manti, see Manty

Mantou 饅頭 186 


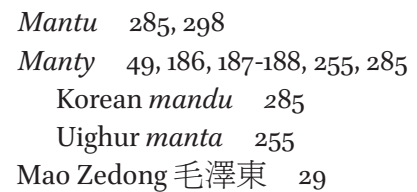

Maotai 茅台 (Chinese liquor) 60

Marco Polo 55, 109, 134, 140, 153-155, 185

And noodles 185

Did get to China 140

On Mongol drinking ritual 153

On Mongol feasting 154-155

Maritime silk roads 291

Marjoram 62

Marmots, as food 159,264

Mead 151

Meat 192-195

Medicine, in Medieval Central Asia 116

Medieval Warm Period (Medieval Climate Anomaly) 12-13, 103, 129, 135

$M e i$ 梅 (Chinese apricot) $\quad 94,156$

Melons 31-32, 57, 163, 174, 181, 205, 221-222, 256

Menander, King of Bactria $\quad 91$

Merv 34

Mice, as food 150

Migration 42

Milindapañha 91

Milk 49, 148, 149, 182, 197-198, 259-26o

Sprinkling $\quad 259-260$

Milk ritual, Mongol, see Tsatsal

Millet $31,49,54,57-59,75,78,81,111,125,148$, 15o, 151, 174, 175, 19o, 206, 235, 281, 283

Broomcorn millet, origins $\quad 57-59$; spread into Central Asia 75

Foxtail millet, origins $\quad 57-58$

In Afghanistan bread $\quad 206$

In early Xinjiang $\quad 78$

Millet ale $\quad 151$

Ming Dynasty $\quad 56,141,161-162,289$

Mint 201

Modernization, concept 204

Mon languages $\quad 71$

Möngke-qan 131-133, 152-153

Tree of life $\quad 152-153$

Mongol China, Arabic Medicine in $\quad$ 145-146

Mongol empire $\quad 83,86,87,125^{-135}, 142-161$

Attacks on North China 130

Conquest of Khwarzmian Empire 130
Cooking 143-144

Food and medicine $\quad 142$

Invasions of Russia and Europe 131

Post-imperial history $\quad 161$

Raiding 129

Rise of and climate 129

"Tengrism," 83

Wars with Jin 130

World Conquest 129

Mongol food 168, 194, 257-267

Boil meat 194

Breads 263

Dairy foods $258-260$

Distilling 168, 26o-261

Dumplings, and noodles $\quad 263$

Fruits and vegetables uncommon $\quad 264$

Gathered foods 266

Herbs 265-266

Jerky 262-263

Meat esteemed food 262

Medical 266-267

Milk tea 259

Modernization 263

Mountain foods 265

Rose hips $\quad 265$

Sausage 262

Soup 263

Tea 259-261

Tsatsal, milk-scattering ritual 259-26o, 267

Whole-cooked lamb $\quad 261$

Wild-gathered foods $\quad 265-267$

Mongolia $1,7,15,16,18,19,26,29,35,40,41$, $44,48,78,170,177,205,257^{-25} 8,264$

Conservation 26

Geography, landscape $\quad 257$

Livestock, five snouts $\quad 264$

Roads, problem of 44

Sacred landscape $\quad 257-258$

Sacred trees $16,18,19$

Mongolian language $\quad 4,70$

Mongols 21, 40-41, 46, 83, 86, 258, 263-265, 267

Belief systems $\quad 267$

Mythic origins $\quad 46$

Nomads 40

Religion 83

Respect for animals $\quad 264-265$ 


\author{
Respect for nature $\quad 263-264$ \\ Sing to livestock $\quad 264$ \\ Subsistence 258 \\ Traditional medicine $\quad 267$ \\ Moti Mahal restaurant, Old Delhi \\ Mulberries 32, 205, 206, 216, 235 \\ In Afghanistan $\quad 206,216$ \\ Staple for mountain Tajiks 235 \\ Mung beans 6o \\ Muraviev, Nikolay 171 \\ Mushrooms, as food 200 \\ Mustard 106 \\ Myrobalans 125
}

212,219

Nan (bread) 93, 174, 182-184, 206, 222, 234, 236,255

Nauruz 52, 217, 230

In Afghanistan, foods 217

Uzbekistan wheat preparation for 230

Near East $\quad 54-55,57$

"Neolithic Diet," 53

New World foods 162, 205

Arrival in Asia 162

Newman, Jacqueline 255

Ni Zan 倪瓚, cookbook by 109

Nigella 202

Nomadic herding, modern 69

Nomadism, types $\quad 46$

Nomads 41, 42, 43, 44-47, 61, 196

And kinship 46-47

And the land 61

Housing 43

Hunting 43

Lifestyle, history of $\quad 47$

Livestock 42

Mobility $\quad 44-45$

Nomads and agrarian empire $\quad 47$

Settlement of 46

Traditional meal use 196

Noodles $\quad 95,96,181,185,194-19$ o, 192, 279, 283

Earliest known $\quad 185$

In China $\quad 185$

In the Western world $\quad 185$

Nutritional and dietary therapy, under the

Mongols 142

Oats $42,173,174,175,279$

Ögödei-qan 35,131
Oil (food) $\quad 148$

Olives 106

Onions $49,95,174,181,199=200,280$

Chinese green 95

Oqol-qaimish 131

Oranges 95, 107-108, 125

Organ meats, and blood 195

Orkhon Inscriptions 90

Ostrovskikh, Peter, on Central Asian food 174

Otrar massacre 130

Ovoo $25^{8}$

Pallas, Peter 164-169

Distillation 164, 165-166, 168

Paklama, Uzbek baklava-like dish $\quad$ 231-233

Palov or Plov $\quad 226$

Pamir Mountains $\quad 7,8$

Pashtun language 73

Pasta 144, 208-209

Afghanistan forms 208-209

Pastoral nomads, and Uighur 5

Pea (European) 105, 173, 235

Peaches 32, 94, 125, 174, 205

And apricots 94

Peanuts 281

Pears 94, 174, 238

Pegolotti 142

Pepper, black $\quad 62,111,125,148,181,162,201$

Long $\quad 125,162$

White 148

Peremech 188-189

Perilla $\quad 284$

Perry, Charles $\quad 187-188,196,203,212,214,217$, 227-229, 240-241, 259-263, 278-279

Persia and Persian Empire 88,90

Persian foodways $5^{2}$

Pickles 211

In Afghanistan 211

Pigeons, domesticated 69

Pigs $65,69,264,280,285$

Domestication 65

Pilaf (polo, pilau) 97, 192-193, 209-210, 226, 231, 251, 255

In Afghanistan, pilau 209-210

In Azerbaijan, plov $\quad 251$

In Uighur culture, polo or palao 255

In Uzbekistan, palov or plov 226, 231 
Pine-nuts $\quad 200,283,284$

Pineapple 281

Pines, bark, cones, and seeds eaten 200

Piora Oscillation $\quad$ 2200-200о BCE $\quad 76$

Pistachios 6o, 106

Pit-cooking 194

Plants, domesticated 56-6o

Platycodon tips as food in Korea $\quad 284$

Pliny the Elder 110

Plums 32, 174, 202

Sour plums 202

Pomegranates $32,60,181,252$

Syrup 252

Poppy seeds 16o, 201

Population of Central Asian nations 31

Portuguese, expansion in 16th century 162

Potatoes 49, 176, 180, 205, 236, 281

Pottery, origin 54

Prehistory 53

Przevalsky Horse $\quad$ 23, 67

Pulses, in Afghanistan 206

Pumpelly, Raphael 37

Pumpkins 176, 236

Qablipilau 210-211

Qaidu Qanate 134

Qan (Turkic term for ruler) defined $\quad 126$

Qanat 35

Qanates, divisions of the Mongol Empire 134, 135

Decline of 135

Qaraqazan 245-246

Qaraqorum 34-35, 90, 131, 149

Qattama 249

Qåwurma Palåw, Samarqand style 227-228

Qimin yaoshu 离民要術 102

Qin 秦, state 97

Dynasty $87-88,97$

Quinces 174

Qing Dynasty $\quad 29,89,170-171,177,178$

Imports of fish and mushrooms $\quad 171$

In Mongolia 170

In Siberia and Central Asia $\quad 171$

In Tibet 170

Qorma-i Sayib 213

Qubilai-qan 24, 55, 133-134, 282

Queen Mother of the West 98

Quick Manta 209
Qurma 193

Qurt (qrut; dried milk solids; aaruul) 152, 197, 236, 241-242

Kazakh production method $\quad 241-242$

Quvud 278

Radish $\quad 284$

Raisins 111

Rashīd al-Dīn 108, 144

Hospital 144

On food 144

Raspberry jam 249

Recipes, see also separate list

Records, problem of 90

Red Current Sharbat $\quad 217$

Regimen 118

Reindeer 46, 264

Religion and Central Asian foodways 82, 288

Religious tolerance in Central Asia $\quad 83-84$

Respect, for nature 43

Rhubarb 148

Rice $\quad 54,58-59,96,107,119,144,174,178$, 209211, 236, 279, 283

In Afghanistan 209-211

In humoral medicine $\quad 119$

Origins $5^{8}$

Richtofen, Ferdinand von 1, 135-136

Rodents eaten in Medieval Mongolia $\quad 150$

Rome and Roman Empire 88

Rosenberger, Nancy $\quad 224-226,229-$

Rouran (Avars) 44. See also Avars

Ruba'iyat of Omar Khayyam 30, 192

Rubruck, William of, see William of Rubruck

Russia and Russians 29, 170-172, 175, 177, 178, 18०, 204, 205, 221, 287

Influences on food $178-180$

Russian language $\quad 72$

Rye $151,173,174,177,235$

Early reference $\quad 151$

Replacing millet as staple $\quad 173$

Sabban, Françoise $\quad 181$

Saffron 106, 182, 202

In Iran 220

Sahlins, Marshall 126

Saiga antelope 23 
Sakas 110

Saksaul 15, 266

Salma 114, 184-185

Salt 148

Samarkand $6,142,162,204$

Heavy eating 142

Samosa 49

In Uzbekistan 229

Samusa 186

Sardāba 35

Sauer, Carl, on domestication 54

Sausage, horse tripe 150

Scapulimancy 163

Scripts and transcriptions 3

Scythians 80,110

Sea buckthorn 18,265

Sea routes, Seattle 289-29o

Sea trade, rise $\quad 289$

Seattle, as modern Silk Road termnius 290291

Seaweeds 284

"Secondary products revolution" 66

Secret History of the Mongols $\quad 13,47,127-128$, 143

Semenov, Pyotr, on food 175

Semirech'ye 81

Sesame $57,60,106,174,181,28$ o, 284

Shams al-D\&n Samarqand $\$ 125$

Shang 㓏 Dynasty 77,87

Shangdu上都 22

Shanyu 單於 (Xiongnu ruler) 47

Sharbat 198-199, 204, 216-217

In Afghanistan 216

Shashlyk 193

Sheep 41-42, 49, 64, 173, 181, 192, 220, 264, 268, 28 o

Domestication and Central Asian forms 64

Fat-tailed 64

Shelpek 249

Shennong Bencao Jing 神農本草經 117

Shilaer 食來兒 254

Shish kebab 214

Shishkabab 193

Shülen 193

Siberia 171, 174

Sichuan, land route to south 140

Signature spicing 201
Silk $\quad 88,135-136$

Roll of on Silk Road, in China 136

Silk Road 1, 52, 88-9, 95, 104, 110, 134-142, 162, 178-179, 289

And food exchanges $\quad 52,95$

Decline and fall 162

History, China $\quad 88,136$

In Mongol period $\quad 135,140$

In Tang 唐 times 104

Influences in Central Asia $\quad$ 178-79

Maritime 110

New Cities 134

Origin of term 1

Products 136

Traffic on 139

Silkworms 136, 144

Sintasha culture $\quad 76$

Sogdian language 73

Sogdians 103

Soju 285-286

Song 宋 Dynasty $105,109,110$

Food 110

Maritime Contacts 109

Sea food 109

Sorghum $31,59,60,108,163,174,175,279-$ 28 o, 283

Soybeans 270

Soy sauce 49,202

Spanish, expansion in 16 th century $\quad 162$ Spelt (variety of wheat) 173

Spices 62, 95 136-137, 141, 142, 215-216, 28o

In China, ones that were adopted and ones that were less so 280

Lists of "spices," including medicinals, on the Silk Road 136-137, 141, 142

Spices and flavorings in Afghanistan 215216

Spices and flavorings, arrival of 62 Spicing of Central Asian food 201-202

Spinach 49, 57, 106, 280

Squashes 174,176

Steppes 14-15

Strawberries 18,112

Sturgeons 114, 220, 252

Sugar, in Afghanistan $\quad 216$

Sugarcane 125

Sui 隋 Dynasty 89,103 
Sü̈̈tei chai, "milk tea," "tea with milk” 259, 26o, 261

Sumac 202, 253

Sweet potatoes $\quad 162,281$

Sweets 200-201, 216, 221-279

Gao Lian's Ming Dynasty sweets recipes 271-279

In Afghanistan $\quad 216$

Syr Darya $\quad$ 9, 32, 34

Taba nan 248

Tabib 144

Tacuinum Sanitatis $\quad 125$

Tajik food 234-237

Dairy food 236

Festival foods 237

Meat 237

Settlements and food 235

Social change 237

Tajikstan $\quad 1,28,125,177$

Languages 235

Talas River, battle of $\quad 88,103-104$

Tamarind $\quad 125$

Tamerlane $\quad 141,161$. See also Timur

Tandur 202-203, 207, 222, 236

Tang 唐 Dynasty $89,102-106,110,140$

Central Asian influence 104

New Plants 105-106

Tang food 110

Tangut Lungs $\quad 232$

Tanistry 127

Tanksūq-nāma-yi Ilḥ̄̄nì dar funūn-i 'ulūm-i Khitāy 147

Tao Hongjing 陶弘景 102

Tarim Basin $\quad 8,12,78$

Mummies $\quad 78$

Tarim River 34, 44

Tashkent $32,60,73,222$

Languages in 73

Public market 222

Tea 107, 122, 144, 181, 198-9, 216, 234, 235, 270

In Afghanistan $\quad 216$

In Uzbekistan 234

New to mountain Tajiks $\quad 236$

Shops 199

Temüjin $\quad 127$

Tengri and "Tengrism," 83
Terracina $\quad 151$

Thai language, word for chicken $\quad 79$

Theophylactus Simocatta 110

Thyme 62

Tianshan 天山 Mountains $\quad 7,8$

Tibet 42,170

Tibetan Buddhism 170

Tibetan language $\quad 71$

Tibeto-Burman languages 71

Timur (Timur Lenk, Tamerlane) $\quad$ 141, 161

Toba Wei 魏 101

Tobacco 162

Toghun-Temür, Mongol emperor 282

Korean wife Ki 282

Tokharian languages $\quad 71$

Tolui-noyon $\quad 131$

Tomatoes $\quad 49,176,180,200,205,236,281$

Transhumance 42

Treaty of Aigun 171

Trebizond 135

Tsatsal (Mongol milk-sprinkling ritual) 149, 259

Turan 86, 90

Turkey $\quad 55,151,176,179,254-255$

Central Asian foods $\quad$ 151, 254-255

Turkic languages $\quad 3,5,49,70,71-72$

Turkic peoples, origin $\quad 46,101$

Mythic origin $\quad 46$

Turkish food, Central Asian roots 254

Turkish language 72

Turkistan 115

Science 115

Turkmenistan $\quad 1,28,34,177$

Turks, emergence of 101

Turmeric $60,125,202$

Turnips 174

Turpan Depression 7, 36

Tutmāj (Medieval bread recipe) 113

Tuva, tombs 80

Uighur food $\quad 255^{-257}$

Ash $\quad 256$

Chinese influences $\quad 256$

Ngan, nan 255

Noodles 255

Polo, pulao 255

Sheep food of choice $\quad 256$

Xinjiang melons 256 
Uighurs $4,5,105,178$

History 5

Languages 5

Religion 5

Ulaanbaatar 37,40

Urban sprawl 37,40

$[U]$ mach 253

United States 28

USSR 29, 37

Uzbek people 5, 221

Uzbek and Kyrgyz, medicinal use of plants 234

Uzbekistan $\quad 1,28,34,37,46,51,59,85,177$, 221, 224-226

Curse of cotton 224

Origins 221

Private plots 225

Urban sprawl in 37,225

Social conditions $\quad 224-226$

Village siting in 37

Uzbekistan, food $\quad$ 95, 221-234

Ancient fruit-growing 95

Baklavas 231-232

Breads and dairy, products, jams,

etc. 233

Dairy 222-223

Dishes 223

Drinks 234

Food and foodways 221-234

Food and the new 230

Food as social indicator $\quad 224-225$

Fruits and nuts 223

Gender structure and food 230

Horse meat 232

Local dishes 223

Marriage 230

Meat 222

Meat and fruit stews 231

Medicinal herbs, wild herbs for medicine and food 234

Nan 222

Palov, kebab and dumpling, and

noddle dishes 233

Palov or plov 226-228

Popular dishes 229-230

Rice 222

Russian and Korean food 230

Soups 223, 233

$$
\begin{array}{ll}
\text { Spicing } & 223 \\
\text { Tandur } & 222 \\
\text { Tripe } & 232
\end{array}
$$

Van Oudenhoven, Frederik, and Jamila Haider, on Tajik food $\quad 234-238$

Vasco da Gama $\quad 161$

Vegetables, in Central Asian cooking 31, 199-200, 211

In Afghanistan 211

Vegetables and spices $\quad 31$

Vegetation, overview 13-20

Vietnam 133

Vinegar 202

"Virgin lands," 16

Visson, Lynn, on Uzbek food $\quad$ 231-233

Volga River 9

Wallerstein, Immanuel $\quad 87$

Walnuts 94, 111, 148, 174, 216, 233, 235-236, 238

In Afghanistan $\quad 216$

In Uzbekistan 223

Warring States period 77

Watermelons $\quad 60,108,163,174,250,256$

Jam 250

Wedding foods, in Afghanistan 217

Weeks, Martha 238

Wei Dynasty 101

Wheat $31,49,54-56,57,59,75,77-78,81,112$, $148,151,173,174,175,181,183-184,191-192$, 220, 235, 279

Bread wheat, origins 57

Einkorn 56

Emmer 57

Foods 191-192

Foods in China 279

In China 279

In early Xinjiang $\quad 78$

In Iran 220

Spread into Central Asia $\quad 75$; to

China 77

Wheat flour 148

Whey 49

Whole animal, cooking $\quad 194$

Wild plants, gathering of 62

William of Rubruck 149-153

On grut making 153 
On Mongol dogs 151

On Mongol food 149-153

Description of liquor fountain $\quad 152$

Williams, Dee Mack 39

Willow Steamed Lamb 194

Wine $115,148,149,151$

Wolfthorn berries, see Goji berries, Lycium spp.

Wolf Soup 24

Wolves 22

Sacred 21-22

Taken with hunting eagles $\quad 22$

Women, power of 48

World-system theory $87,88,89$

Wulff, Hans 218-219

Xia 夏 kingdom $\quad 76$

Xianbei 70,101

Xinjiang Province $\quad 1,4-6,12,36,41,52,72,76$, $78,84,96-97,170,174,178,181$

And China 6

And religions 5

And Uighurs 4

Chinese migration 4

Early towns $\quad 76$

Food 96

Han settlers outnumber Uighurs $\quad 178$

Xiongnu $42,47,70,8$ o, 88, 97

Yaks in 42

Xixia 西夏, state 110,131

Xuanzang 玄牀, monk 104, 111

Yaks $\quad 41-42,49,66,264$

Yamnaya culture $\quad 76$

Yaqub Beg 170

Yellow sapote 281

Yesügei 127

Yinshan zhengyao 飲膳正要 $24,153,155$, 160,184

Game recipes 24

Noodles 184

Yin zhuan fushi lian 飲饌服食箋 271

Yogurt 49

Yuan Dynasty $\quad 86,89,102,289$

Yuezhi 月支 86

Yurt 43. See also Ger

Zakhïra-i-Khwārazmshāhī 145
Zhang Qian 張騫 $\quad$ 97-98

Zhongdu 中都 130

Zhou 周 Dynasty 77,87

\section{List of Recipes}

\section{Afghanistan}

Dopiaza, stew with onions $\quad 215$

Egg bread 208

Nauring palaw, pilau with oranges $\quad 213-214$

Pilau, types and recipes 209-210

Qablipilau 210-211

Qorma-i-Sayb, meat with apples and split peas 213

Quick manta 209

Jujia biyong shilei (Medieval household

encyclopedia)

Baldy 158

Güllach 158

Halwa $15^{8}$

Julapia 159

Kogurma 157

Qarisa 160

Quresh-e 158

Rolled thin pancakes $\quad 156$

Sour soup 157

Sweet börek 156

Tutum-ash 157

West of the River Lungs $\quad$ 16o

\section{Kazakh}

Bauyrsaq, Kazakh doughnuts 246-247

Melon jam 250

Qattama, puff pastry 249

Raspberry jam 249

Shelpek, fried thin bread 249

Taba nan, bread 248

Turniyaz, soup with qurt 241

Watermelon jam 250

Medieval Syrian, noodles

Salma 113

Tutmāj 113

Ming China, Gao Lian's recipes

Black and moist candy $\quad 276$ 
Comb-print crisps $\quad 275$

Crisp cookies $\quad 277$

Flour-with-oil candies 273

Ghee 273

Goat "marrow" candy $\quad 276$

Manchu candied fritter 274

Nest of threads candy 275

Pepper-and-salt cookies $\quad 276-277$

Pine nut cookies 273

Pine nut halva $\quad 273-274$

Puffed buckwheat $\quad 275^{-27} 6$

Roasted flour 272-273

Saboni $\quad 276$

Shilaier, sweet 254

Slices with mint (candy) $\quad 275$

Snowflake shortbread $\quad 274$

Sugar syrup 272

White and moist candy $\quad 274$

Wind-dissolved cakes 277

Yellow and moist halva $\quad 274$

Tajik

Halva $\quad 278$
Tatar

Peremech dumplings 188

\section{Uzbekistan}

Kok chuchwara, green dumplings 228-229

Oshkovok manti 187

Qawurma palaw, meat with rice $\quad 227-228$

Yinshan Zhengyao (Medieval Mongol)

Bal-po soup 193-194

Barley samsa $\quad 189$

Barley soup (harisa) $\quad 252$

Cherry concentrate $\quad 252$

Chuqmin, "long bread," 207

Cut flowers manta $\quad 187$

Pomegranate syrup $\quad 25^{2}$

Poppy seed rolls 160

Red currant sharbat 217

Roast wolf 24

Tangut lungs $\quad$ 232-233

Umach noodles 253

Willow-steamed lamb $\quad 194$ 